\title{
Radiative transfer model STORM for full Stokes vector calculations in the visible and near infrared spectral range
}

\author{
U. Böttger ${ }^{1}$ and R. Preusker ${ }^{2}$ \\ ${ }^{1}$ DLR, Institut für Planetenforschung, Berlin, Germany \\ ${ }^{2}$ Institut für Weltraumwissenschaften, Freie Universität Berlin, Germany
}

\begin{abstract}
Based on the Matrix-Operator Method the radiative transfer code STORM (STOkes vector Radiative transfer Model) is introduced, which was developed in a joint project of DLR and Institut für Weltraumwissenschaften-Freie Universität Berlin. STORM calculates the Stokes parameters $(I, Q, U, V)$ in a plane parallel, multi layered atmosphere in the visible and near infrared spectral range. The scattering characteristics of aerosols are determined by Mie theory. The surface represents a Lambertian reflector or a wind ruffled water surface described by Cox-Munk model. The results of one calculation are the upward and downward directed Stokes parameters for one wavelength at a desired number of sun incident and viewing angles at varying altitudes in the principal plane and other azimuth angles. STORM is applied for an analysis in view of designing downward looking Earth observing optical remote sensing systems and values of the degree of polarization are presented as generic basis for remote sensing system design and data processing.
\end{abstract}

\section{Introduction}

Polarization of light passing an atmosphere-surface system affects the measurements of earth observing optical instruments. There are several possibilities to care for this effect. One is to measure the polarization of the incoming radiation. This allows the use of additional information for better retrieval of parameters of the observed system (Herman et al., 1997; Leroy et al., 1997; Deuze et al., 2001a,b; Goloub et al., 1999). Another possibility is to correct the polarization sensitivity by optical polarization scrambler systems (GOME, 1995). But one can also study the observed systems to find out if polarization is significant for the desired measurements. This was the motivation of this study for the design of airborne spectrometers to provide an insight into

Correspondence to: U. Böttger (Ute.Boettger@dlr.de) the polarization variability of the radiation due to effects of atmosphere and surface. This allows the estimation of a maximum acceptable instrumental polarization sensitivity to ensure that the polarization uncertainty of the measurements is below a critical limit.

In this paper the radiative transfer code STORM (STOkes vector Radiative transfer Model) to solve the radiative transfer equation for the full Stokes vector for varying flight altitudes, wavelengths and viewing geometry is introduced. The radiative transfer code considers Lambertian and specular reflecting surfaces. The concept of a Lambertian surface stands for fully depolarizing reflection, whereas a specular reflecting surface polarizes the incident light in dependence on viewing geometry and reaches full polarization at the Brewster angle. With these two concepts the extreme cases of polarization reflection features of land and water surfaces have been considered, covering their full variability. Different aerosol types and a pure Rayleigh atmosphere are considered.

\section{Polarization of light in an atmosphere-surface system}

Radiation in the visible and near infrared spectral region is considerably polarized (Coulson, 1988). The Stokes parameters $(I, Q, U, V)$ are used to describe the full state of radiation (Chandrasekhar, 1950): $I$ is the intensity. $Q, U$ describe the part of linear polarized light and the plane of polarization. $V$ discriminates between linear, elliptic or circular polarization. $\left(I_{l}, I_{r}, U, V\right)$ is another widely used description with $I=I_{l}+I_{r}$ and $Q=I_{l}-I_{r}$ where $l$ and $r$ stand for parallel and perpendicular to a reference plane component. The degree of polarization is defined as

$P_{\mathrm{deg}}=\frac{\sqrt{Q^{2}+U^{2}+V^{2}}}{I}$

Published by Copernicus GmbH on behalf of the URSI Landesausschuss in der Bundesrepublik Deutschland e.V. 
The plane of polarization $\chi$ is defined as

$\tan 2 \chi=\frac{U}{Q}$

Polarization is caused by interaction of the incoming unpolarized solar irradiance with the atmosphere and the surface. The important processes causing polarization of light in nature are scattering at gas, aerosol and cloud particles in the atmosphere and reflection at soil, sand, vegetation, water and other surface types.

The degree of polarization in the atmosphere depends in a very complex way on the various atmospheric parameters like Rayleigh and aerosol optical thickness, aerosol type and their spectral properties (Breon et al., 1995; Deuze et al., $2001 b$,a). Light single scattered at Rayleigh particles is fully polarized at $90^{\circ}$ scattering angle despite of a small depolarization factor (Chandrasekhar, 1950). Multiple scattering processes depend directly on optical thickness (Iqbal, 1983) and decrease the maximum value of the degree of polarization without changing its viewing angle position for a specific sun incident angle. Non-absorbing or weakly absorbing aerosols have a depolarizing effect on the maximum of the degree of polarization of a Rayleigh atmosphere. Additional neutral points can be observed. The maximum degree of polarization decreases continuously from the top of atmosphere (TOA) to lower flight altitudes. This behaviour is independent of the sun incident angle. The degree of polarization of an aerosol loaded atmosphere can increase in specific cases to a maximum of $70 \%$ but ranges in dependence on wavelength and viewing geometry normally around $35 \%$.

The plane of polarization defined by the angle $\chi$ (Eq. 2) is relatively independent of wavelength and Lambert surface reflection (Coulson, 1988). The only exception is the region of neutral points, where $\chi$ undergoes a sign change. Multiple scattering has little effect on the plane of polarization in contrary to the noticeable effect on the degree of polarization.

If gaseous absorption must be taken into account (e.g. in the spectral range of the $O_{2}-A$ band around $760 \mathrm{~nm}$ ), the relative change (increase or decrease) of the degree of polarization compared to the absorption-free spectral range near this band depends on the absolute value of the gas absorption as well as on the flight altitude and the height distribution of aerosols and gases in the atmosphere (Preusker et al., 1995). Normally the degree of polarization increases with increasing absorption due to the decrease of multiple scattering and tend towards a Rayleigh like single scattering behaviour.

Natural surfaces are a complex mixture of different materials in all three phases (solid, liquid, gaseous). The interaction of electromagnetic radiation with natural surfaces is characterized by texture, chemical and mineral compounds, moisture and density (Breon et al., 1995; Coulson, 1988; Egan, 1985). Unpolarized light reflected by mineral surfaces (sand and other soils) is partially polarized and the degree of polarization ranges between $0 \%$ and maximum $40 \%$.
The polarized part of light reflected by vegetation is caused by specular reflection at the surfaces of the leafs (Vanderbilt and Grant, 1985; Breon et al., 1995). For most conditions the values are below $30 \%$ (Vanderbilt, 2001).

Light incident on water surfaces is reflected and transmitted according to Fresnel's law and polarized in dependence on incident angle. The maximum degree of polarization is reached at the Brewster angle, which is about $53^{\circ}$ for water. Directly above a wind ruffled water surface two maxima of the degree of polarization occur at the Brewster angle (in forward and backscattering direction) that can reach about $100 \%$. At flight altitudes larger than or equal to $3000 \mathrm{~m}$ in forward scattering direction two other maxima of the degree of polarization (specular reflection at water surface and Rayleigh scattering of the atmosphere) superpose. The degree of polarization decreases with increasing flight altitude or remains unchanged in dependence on aerosol type and optical thickness and reaches values larger than $90 \%$.

Light reflected at a Lambertian surface is unpolarized, that means Lambertian reflection does not produce polarization and fully depolarize incident light. The degree of polarization at the top of an atmosphere-Lambertian-surface-system is greater than zero due to the atmospheric influence. The maximum degree of polarization decreases from top of atmosphere to lower flight altitudes for an aerosol loaded atmosphere with low Lambertian surface reflection or with no surface reflection. The degree of polarization reaches maximum values of $45 \%$ and medium values of $<15 \%$ for aerosol loaded atmospheres above a surface of an albedo of 0.05 . Increasing surface albedo reduces the degree of polarization at all viewing angles and all flight altitudes drastically.

\section{Radiative transfer method used in STORM}

The radiation field in the atmosphere is determined by single and multiple scattering at air molecules, aerosols and droplets and by interaction with the surface. Starting point for the investigation of single and multiple scattering effects in an atmosphere-surface system is the radiative transfer equation (Chandrasekhar, 1950)

$$
\begin{aligned}
& \left(\mu \frac{d}{d \tau}+1\right) I(\tau, \mu, \phi)=\pi \varpi_{0}(\tau) \widetilde{P}\left(\mu, \mu_{0}, \phi, \phi_{0}\right) S_{0} e^{-\frac{\tau}{\mu_{0}}} \\
& +\varpi_{0}(\tau) \int_{0}^{2 \pi} \int_{-1}^{+1} \widetilde{P}\left(\mu, \phi, \mu^{\prime}, \phi^{\prime}\right) I\left(\tau, \mu^{\prime}, \phi^{\prime}\right) d \mu^{\prime} d \phi^{\prime}
\end{aligned}
$$

with $\mu=\cos \theta$. Here $I(\tau, \mu, \phi)$ is the full Stokes vector of the diffuse scattered radiation field at boundary $\tau$. $\theta$ and $\phi$ are the zenith and azimuth angles, respectively. $S_{0}$ is the spectral solar flux incident at the top of atmosphere, $\mu_{0}$ and $\phi_{0}$ are the cosine of zenith angle and azimuth of incident solar flux, $\mu$ and $\phi$ are the corresponding values in viewing direction. $\varpi_{0}(\tau)$ is the single scattering albedo of the scatterer in the considered layer. $\widetilde{P}$ is the phase matrix rotated into the incident and viewing planes. This presentation of the radiative 


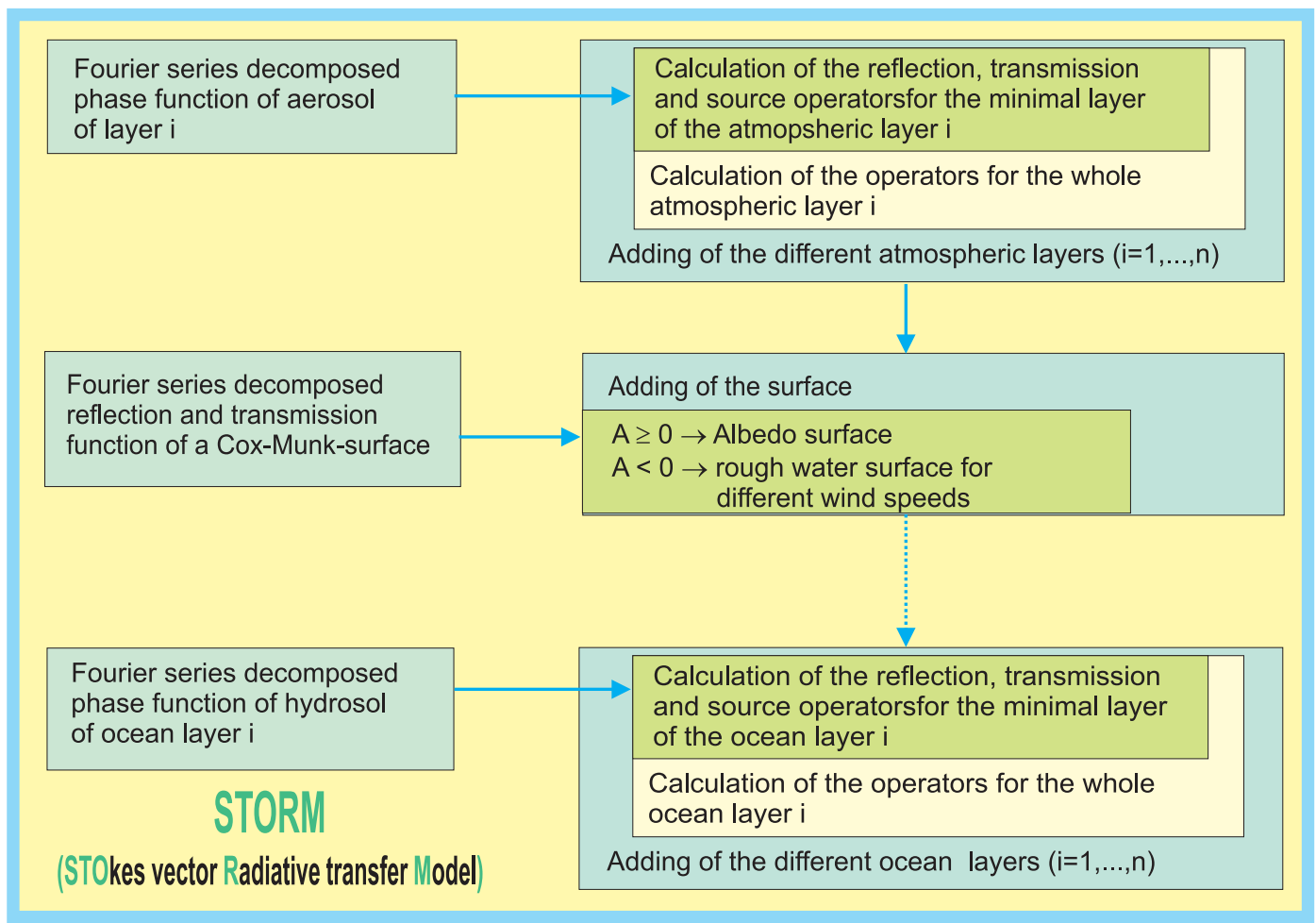

Fig. 1. Scheme of the radiative transfer simulation code STORM for an atmosphere-ocean system. Solution of the radiative transfer equation with the Matrix-Operator-Method.

transfer equation is valid for plane parallel atmospheres. A plane parallel vertically inhomogeneous atmosphere-surface system can be approximated by a number of homogeneous layers. The local dependence is described only by the optical thickness $\tau$, which is an integral value of extinction over flight altitude. Refractive characteristics of the atmosphere are not considered. The first term on the right side in Eq. 3 describes the first or single scattering process of the radiation in the atmosphere. The second term describes multiple scattering and includes all following scattering processes, that contribute to the Stokes vector $I$ into direction $(\mu, \phi)$.

To solve the radiative transfer equation for a plane parallel inhomogeneous atmosphere the matrix operator method is used (Plass et al., 1973). The basis of this method is the interaction principle applied to the transmission, reflection and source operators for a single layer, which relates the upward and downward directed incident and emerging radiation and the sources of this layer with each other. These operators are retrieved by a coefficient comparison of the radiative transfer equation written in matrix form with the interaction principle for infinitesimal layers. The application of the general interaction principle determines the transmission, reflection and source operators for the whole atmosphere-surface system, and the diffuse upward and downward Stokes vectors at the top and bottom of the atmosphere and at every internal layer. So the reflection, transmission and source operators can be calculated for the whole atmosphere-surface system and the upward and downward diffuse radiation field can be derived at every boundary in vertically inhomogeneous atmospheres.

The surface is treated here as an additional layer with special reflection operators and no transmission. For an isotropically reflecting surface the model of a Lambertian reflector is applied, which transforms any incident radiation distribution into a uniform unpolarized distribution. The reflection at a wind ruffled water surface is described by Fresnel's law combined with a surface wind speed dependent probability distribution of the slope of the water surface developed by Cox and Munk (1954).

Based on the Matrix-Operator Method the radiative transfer code STORM (STOkes vector Radiative transfer Model) (Fig. 1) was developed in a joint project of DLR and Institut für Weltraumwissenschaften - Freie Universität Berlin (Böttger, 1997; Fell and Fischer, 2001). STORM calculates the Stokes parameters $(I, Q, U, V)$ in a plane parallel, multi layered atmosphere. The scattering characteristics of aerosols are determined by Mie theory. The surface represents a Lambertian reflector or a wind ruffled water surface. The results of one calculation are the upward and downward directed Stokes parameters $(I, Q, U, V)$ for one wavelength at a desired number of sun incident and viewing angles at varying flight altitudes in the principal plane and other azimuth angles. A comparison of the Stokes parameters with 
values calculated analytically and tabulated by K.L. Coulson (1960) for a Rayleigh atmosphere for different values of aerosol optical thickness and surface albedo gave an accuracy of STORM much better than $1 \%$ and only for large sun incident and viewing angles better than $2 \%$.

\section{Flight altitude dependence of the degree of polarization}

To describe the polarization at varying flight altitudes it is necessary to perform radiative transfer calculations of the Stokes parameters as there is no measured data available to analyze the flight altitude dependence systematically. STORM is used to perform these calculations. The Stokes parameters are calculated for 13 sun incident and viewing angles at Gauss-Lobatto-quadrature points, which is considered as sufficient to guarantee an acceptable accuracy for the purpose of this paper. The atmosphere consists of a boundary layer $(0 \mathrm{~km}-2 \mathrm{~km})$ containing urban, continental or maritime aerosol types. Above the boundary layer $(2 \mathrm{~km}$ $-12 \mathrm{~km}$ ) continental background aerosol is chosen as tropospheric aerosol. The upper layer $(12 \mathrm{~km}-90 \mathrm{~km})$ contains a background stratospheric aerosol. The layering corresponds to the proposal of WCP (1986). The surface is described by a Lambertian reflector and by a wind ruffled water surface.

As this study is performed for downward looking Earth observing airborne optical sensors some parameters are fixed consciously. To cover the visible and near infrared spectral range and to save computing time two wavelengths $443 \mathrm{~nm}$ and $865 \mathrm{~nm}$ are chosen to represent the visible and near infrared spectral range.

The field of view (FOV) is taken between $\pm 50^{\circ}$, which covers the viewing angle range of optical sensors sufficiently. Only values within this viewing angle range are analyzed.

Five boundary layer aerosol types (maritime $70 \%$ and $99 \%$ humidity, continental, urban $80 \%$ humidity and a mixture of continental and maritime $99 \%$ humidity) are considered for different boundary layer aerosol optical thickness. So nine cases of aerosol loaded atmosphere and one pure Rayleigh case are included into the analysis.

The sensor altitude is fixed at $0 \mathrm{~km}, 3 \mathrm{~km}, 5 \mathrm{~km}, 7 \mathrm{~km}$ and at top of atmosphere, representing typical situations for airborne and satellite remote sensing systems. The surface albedos of $0 \%$ and $20 \%$ cover the range of urban and rural isotropically reflecting areas. With an albedo of $0 \%$ the strongest polarization of the atmosphere is modelled. A wind speed of $6 \mathrm{~m} / \mathrm{s}$ are chosen for a wind ruffled water surface to represent the case of extreme anisotropic reflection.

The variability of the degree of polarization with flight altitude, $P_{\operatorname{deg}}(\theta)$, is analyzed investigating the dependence on viewing geometry, on surface type, on boundary layer aerosol type and optical thickness. Most attention is concentrated on the study of the behavior of the maximum value of the degree of polarization $P_{\max }$, to illustrate the worst case scenario. Linear polarization is assumed as circular polarization can be neglected for the cases considered here.

In Fig. 2 the upward degree of polarization is shown in the principal plane in dependence on viewing zenith angle and flight altitude for $\lambda=865 \mathrm{~nm}$. The principal plane is chosen, because for these azimuths the viewing angle dependence of $P_{\operatorname{deg}}(\theta)$ shows the strongest features and largest values. The albedo is 0 (upper two graphs) and .2 (middle two graphs) and a wind ruffled water surface $6 \mathrm{~m} / \mathrm{s}$ (lower two graphs). The graphs in the right column correspond to a pure Rayleigh case. The left column corresponds to an aerosol loaded atmosphere. As there is no surface contribution in for albedo 0 (upper graphs) the pure atmospheric polarization can be studied. The angular position of $P_{\max }$ can be seen more or less pronounced for all cases around the scattering angle of $90^{\circ}$. For the Rayleigh case (right graph) the highest value of the degree of polarization is reached. Because of the increase of total optical thickness and because of the different scattering characteristics of aerosols and Rayleigh particles $P_{\max }$ decreases if non-absorbing or weakly absorbing aerosols are contributed regardless of the aerosol type. For azimuth $\phi=180^{\circ}$ the degree of polarization shows lower values than for $\phi=0^{\circ}$. Neutral points can be observed. The values of $P_{\operatorname{deg}}(\theta)$ between the neutral points depend on aerosol type and aerosol optical thickness.

For $\phi=0^{\circ}$ the value of $P_{\max }$ decreases continuously from the top of atmosphere (TOA) to lower flight altitudes for all aerosol loaded atmospheres. This behaviour is independent of the sun incident angle. The decrease is due to the relative decrease of Rayleigh scattering (i.e. Rayleigh optical thickness) compared to aerosol scattering contribution (i.e. aerosol optical thickness) with decreasing flight altitude and depends on aerosol type and on aerosol optical thickness. Values of $P_{\max }$ of $50 \%$ can be reached, excluding the Rayleigh case. For the pure Rayleigh case almost no change $(443 \mathrm{~nm})$ or an increase $(865 \mathrm{~nm})$ of the degree of polarization for lower flight altitudes can be observed (no graph). The increase can be explained with the decreasing Rayleigh optical thickness, leading to lower multiple scattering contributions. This is important to take into account if measurements are made at very clear atmospheric conditions. In the backward scattering viewing angle region $\left(\phi=180^{\circ}\right)$ the degree of polarization changes less with flight altitude and the value of the maximum degree of polarization is much smaller than for $\phi=0^{\circ}$. Some of the cases show a slight increase with decreasing flight altitude, and values of $17 \%$ can be reached at $3000 \mathrm{~m}$.

For Lambertian surface with an albedo of $20 \%$ (middle graphs) the contribution due to surface reflection leads to a strong decrease of $P_{\operatorname{deg}}(\theta)$ at all viewing angles. The continuous decrease of the value of $P_{\max }$ from the top of atmosphere (TOA) to lower flight altitude is very small but exists. The mentioned behavior due to surface albedo is independent of sun incident angle, wavelength and aerosol type.

The behavior of the degree of polarization changes sig- 

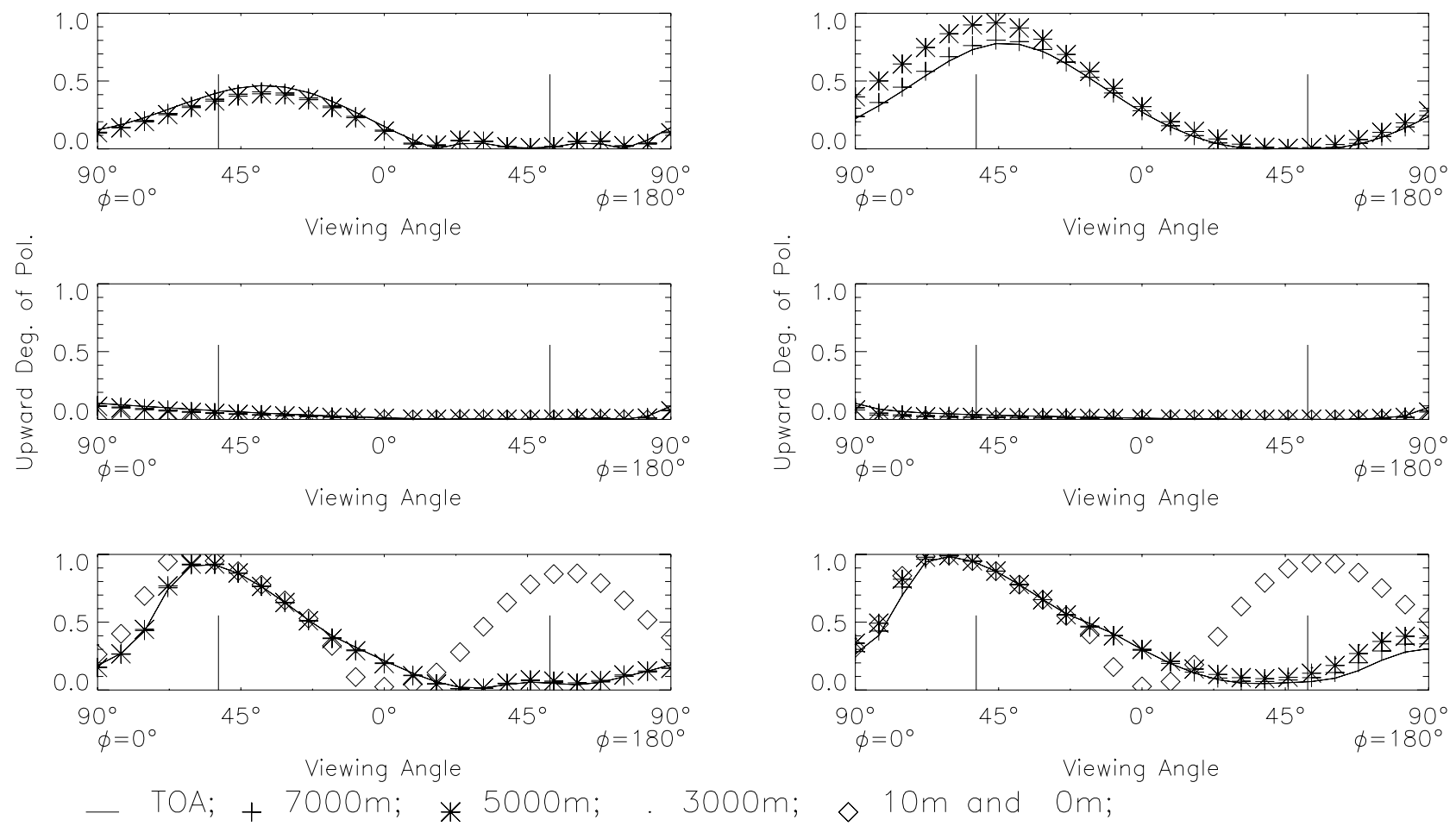

Fig. 2. Upward degree of polarization, $P_{\mathrm{deg}}(\theta)$, in dependence on viewing angle for different flight altitudes. The abscissa is the viewing angle [in degree] in the principal plane $\left(\phi=0^{\circ}\right.$ and $\phi=180^{\circ}$ and $\phi_{0}=0^{\circ}$ ) at $865 \mathrm{~nm}$ and a sun incident angle of $44^{\circ}$. The albedo is 0 (upper two graphs), .2 (middle two graphs) and a wind ruffled water surface $6 \mathrm{~m} / \mathrm{s}$ (lower two graphs). $0^{\circ}$ corresponds to nadir looking. $\phi=0^{\circ}$ means to look into forward scattering (over water into specular reflection) direction, $\phi=180^{\circ}$ means to have the sun in the back while downward looking. Right column corresponds to Rayleigh case. Left column corresponds to an aerosol loaded atmosphere. Vertical bars indicate the field of view of $\pm 50^{\circ}$.

nificantly, if a wind ruffled water surface, as an extreme anisotropic reflection, is considered (lower graphs). For a wind ruffled water surface (here with a wind speed of $6 \mathrm{~m} / \mathrm{s}$ ) two maxima of the degree of polarization above surface and at $10 \mathrm{~m}$ flight altitude are seen. The position of these maxima corresponds to the Brewster angle at which a maximum degree of polarization is expected. Because of the surface roughness and reflected skylight this maximum occurs also in backward direction $\left(\phi=180^{\circ}\right)$. This bidirectional behavior of $P_{\operatorname{deg}}(\theta)$ doesn't change significantly if the surface wind speed is $2 \mathrm{~m} / \mathrm{s}$ (not shown). At $3000 \mathrm{~m}$ flight altitude the surface degree of polarization is masked by the atmosphere. The backward maximum of the degree of polarization disappears, since surface reflected radiance is much lower than the scattered atmospheric radiation and so the atmospheric polarization is dominant. The atmospheric maximum at $90^{\circ}$ scattering angle occurs and is superposing with the maximum at the Brewster angle. Large sun incident angles thus show the phenomenon of two maxima of the degree of polarization for $\phi=0^{\circ}$.

Compared to the cases with Lambertian reflection and no reflection the wind ruffled water surface can lead to high values of the degree of polarization at all flight altitudes for $\phi=0^{\circ}$. The values and the slope of the maximum polarization depend on aerosol type and on aerosol optical thickness. Values of the degree of polarization between $10 \%$ and 90\% are reached for flight altitudes between top of atmosphere and $3000 \mathrm{~m}$. Directly above surface $(0 \mathrm{~m}$ and $10 \mathrm{~m})$ the value of $P_{\max }$ reaches the highest values of more than $90 \%$, which is also true for $\phi=180^{\circ}$. Hence if no information about the boundary layer aerosol is available, high values of the degree of polarization must be assumed. In backward scattering direction the values of the maximum degree of polarization show a slight increase or no change from top of atmosphere to $3000 \mathrm{~m}$. The values range between $2 \%$ and $20 \%$ in dependence on aerosol type and aerosol optical thickness. The dependence of $P_{\max }$ on total optical thickness is shown in Fig. 3. The change of total optical thickness is simulated changing the boundary layer aerosol optical thickness. For a black albedo (upper graph) the decrease of $P_{\max }$ with increasing optical thickness is obvious. The explanation is, as mentioned already above, the increase of multiple scattering processes with increasing optical thickness which leads to a decrease of the maximum degree of polarization. This changes if the albedo is increased to 0.2 (middle part of the figure). For small values of the optical thickness the de- 

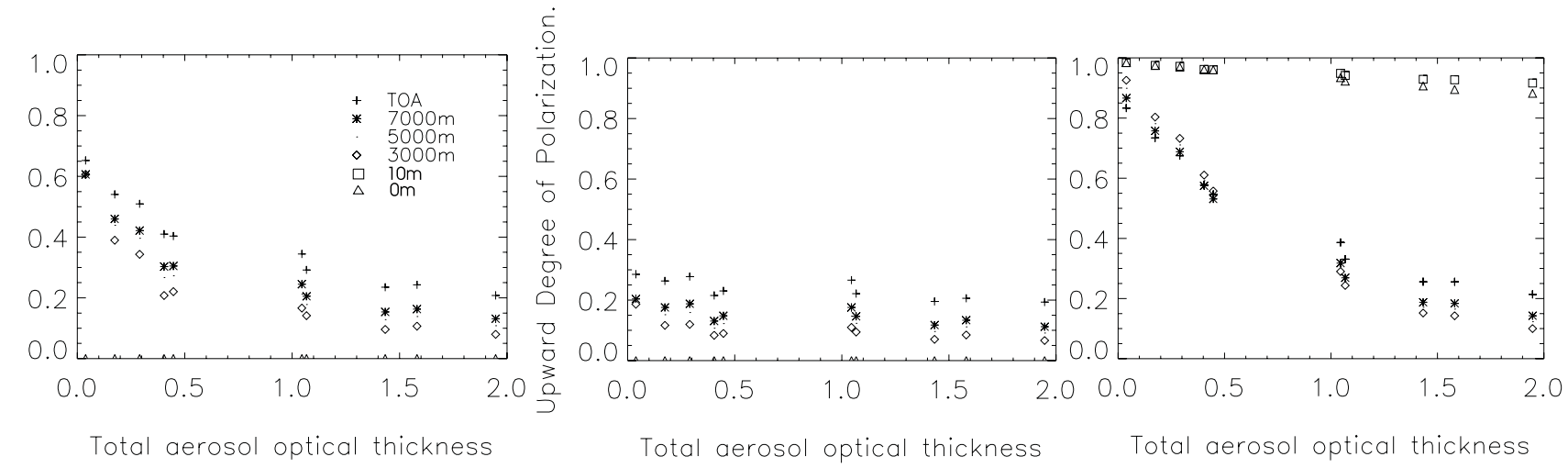

Fig. 3. Maximum value of the degree of polarization in dependence on total optical thickness for different flight altitudes. Left plot corresponds to an albedo of 0 , the middle plot corresponds to an albedo of 0.2 and the right plot corresponds to a wind ruffled water surface with surface wind speed of $6 \mathrm{~m} / \mathrm{s}$. The wavelength is $443 \mathrm{~nm}$.

crease of the degree of polarization is pronounced compared to the case of zero surface albedo, whereas for large optical thickness this decrease is very small. Thus the effect of surface albedo on $P_{\max }$ is larger for small optical thickness and vanishes with increasing optical thickness. This is interesting to notice for measurements in the near infrared spectral range above urban or arid regions that can be described by Lambertian reflection. As most of such surface types show an increase of reflection with increasing wavelength one can expect that the degree of polarization reaches only small values regardless that the optical thickness decreases with increasing wavelength. If a rough water surface is present the aerosol optical thickness has a large influence on the value of $P_{\max }$ (lower graph) from top of atmosphere to $3000 \mathrm{~m}$. Small optical thickness corresponds to large values of the degree of polarization. With increasing optical thickness the value of $P_{\max }$ decreases and reaches values compared to the case with no or Lambertian reflection. Directly above surface, the influence of the optical thickness on the degree of polarization is low. Even for large optical thickness, the value of the degree of polarization is about $90 \%$ directly above surface.

\section{Summary}

The radiative transfer code STORM is used to analyze the height dependence of the degree of polarization at several wavelength and viewing geometries. Maximum values of the degree of polarization occur at scattering angles of $90^{\circ}$ for all wavelengths, flight altitudes, aerosol types and optical thickness.

Pure Rayleigh atmospheres with no Lambertian reflection show the highest values of the degree of polarization. Values of the degree of polarization are larger for $\phi=0^{\circ}$ than for $\phi=180^{\circ}$. The maximum degree of polarization decreases from TOA to lower flight altitudes for aerosol loaded atmospheres with no surface reflection or Lambertian sur- face reflection. The degree of polarization reaches values of $55 \%$ for $\phi=0^{\circ}$ and less than $20 \%$ for $\phi=180^{\circ}$ for aerosol loaded atmospheres above a black surface. Increasing surface albedo reduces the degree of polarization at all viewing angles and all flight altitudes. This effect is strongest for atmospheric conditions with little aerosol load.

Above wind ruffled water surfaces two maxima of the degree of polarization superpose at flight altitudes larger than $3000 \mathrm{~m}$ for $\phi=0^{\circ}$. The degree of polarization decreases with increasing flight altitude or remains unchanged in dependence on aerosol type and optical thickness. It reaches values larger than $90 \%$. Directly above a wind ruffled water surface two maxima at the Brewster angle (for $\phi=0^{\circ}$ and $\phi=180^{\circ}$ ) occur.

For small optical thickness the maximum degree of polarization depends on surface type. For large optical thickness the degree of polarization is almost independent of surface and aerosol type.

Summarizing, the flight altitudes dependence of the degree of polarization is determined by surface and aerosol type and aerosol optical thickness. In order to estimate the maximum acceptable instrumental polarization sensitivity, it is, therefore, important to check and to define the targets of interest (surfaces to be observed) and the measurement conditions (sun incident angle during measurement, atmospheric conditions) carefully. In this way reasonable assumptions of expected values of the degree of polarization can be made.

\section{References}

Böttger, U.: Zur Ableitbarkeit von Aerosoleigenschaften aus Polarisationmessungen, Strahlung in Atmosphäre und Ozean, Beiträge zur Fernerkundung 8, Institut fr Weltraumwissenschaften, Freie Universität Berlin, 1997.

Breon, F., Tanre, D., Lecomte, P., and Herman, M.: Polarized Reflectance of Bare Soils and Vegetation: Measurements and Mod- 
els, IEEE Transactions on Geoscience and Remote Sensing, 33, 487-499, 1995.

Chandrasekhar, S.: Radiative Transfer, Oxford University Press, 1950.

Coulson, K.: Polarization and Intensity of Light in the Atmosphere, A.Deepak Publ., Hampton, Virginia, USA, 1988.

Cox, C. and Munk, W.: Measurements of the roughness of the Sea Surface from Photographs of the Sun's Glitter, J. Opt. Soc. Am., 44, 838-850, 1954.

Deuze, J. L., Breon, F., Devaux, C., Goloub, P., Herman, M., Lafrance, B., Maignan, F., Marchand, A., Nadal, F., and Tanre, D.: Remote sensing of aerosols over land surfaces from POLDER-ADEOS-1 polarized measurements, J. Geophys. Res., 106, 4913-4926, 2001a.

Deuze, J. L., Goloub, P., Herman, M., Marchand, A., Perry, G., Susana, S., and Tanre, D.: Estimate of the aerosol properties over the ocean with POLDER, J. Geophys. Res., 106, 4913-4926, 2001b.

Egan, W.: Photometry and Polarization in Remote Sensing, Elsevier, New York, USA, 1985.

Fell, F. and Fischer, J.: Numerical simulation of the light field in the atmosphere-ocean system using the matrix-operator method, 3, 351-388, 2001.

Goloub, P., Tanre, D., Deuze, J., Herman, M., Marchand, A., and Breon, F.: Polarized Reflectance of Bare Soils and Vegetation: Measurements and Models, IEEE Transactions on Geoscience and Remote Sensing, 37, 1586-1596, 1999.

GOME: User Manual, Publication ESA SP-1182, ESA/ESTEC, 1995.
Herman, M., Deuze, J. L., Devaux, C., Goloub, P., Breon, F. M., and Tanre, D.: Remote sensing of aerosols over land surfaces including polarization measurements and application to POLDER measurements, J. Geophys. Res., 102, 17 039-17 049, 1997.

Iqbal, M.: An Introduction to Soalr Radiation, Academic Press, 1983.

Coulson, K. L., Dave, J. V., and Sekera, Z.: Tables Related to Radiation Emerging from a Planetary Atmosphere with Rayleigh scattering, Univ. of. Calif. Press, USA, 1960.

Leroy, M., Deuze, J. L., Breon, F. M., Hautecoeur, O., Herman, M., Buriez, J., Tanre, D., Bouffies, S., Chazette, P., and Roujean, J.: Retrieval of atmospheric properties and land surface bidirectional reflectances over land from POLDER/ADEOS, J. Geophys. Res., 102, 17 023-17 037, 1997.

Plass, G. N., Kattawar, G. W., and Catchings, F. E.: Matrix Operator Theory of Radiative Transfer. 1: Rayleigh Scattering, Appl. Opt., 12, 314-329, 1973.

Preusker, R., Böttger, U., and Fischer, J.: Spectral and Bidirectional Measurements of the Stokes-Vector in the $\mathrm{O}_{2}$-A Band and Their Interpretation, SPIE, 2582, 13-20, 1995.

Vanderbilt, V. C.: (personal communication), 2001.

Vanderbilt, V. C. and Grant, L.: Plant canopy specular reflectance model, IEEE Transactions on Geoscience and Remote Sensing, 23, 722-730, 1985.

WCP: A preliminary cloudless standard atmosphere for radiation computation, Tech. rep., WCP-Report 112, WMO/TD-No.24, 1986. 